\title{
DOSSIER
}

\section{Los desafíos del racismo a la gestión, docencia, investigación y vinculación social en la educación superior}

\author{
The Challenges of Racism to Management, Teaching, \\ Research and Social Engagement in Higher Education \\ Daniel Mato \\ Consejo Nacional de Investigaciones Científicas y Técnicas (conicet), Argentina \\ Cátedra Unesco Educación Superior y Pueblos Indígenas y Afrodescendientes en América Latina \\ Centro Interdisciplinario de Estudios Avanzados (ciea), Argentina \\ Universidad Nacional de Tres de Febrero (untref), Argentina \\ dmato@untref.edu.ar
}

2

\section{RESUMEN}

El racismo es una ideología según la cual los seres humanos seríamos clasificables en "razas", algunas de las cuales serían superiores a otras. En América Latina, esta ideología, originada en el periodo colonial, aún impregna las maneras de ver el mundo de buena parte de la población, y sus consecuencias afectan especialmente a las personas y comunidades de pueblos indígenas y afrodescendientes.

Y aunque en algunos países "latinoamericanos" (expresión que cuanto menos silencia la existencia de pueblos indígenas y afrodescendientes $)^{1}$ los comportamientos abiertamente discriminatorios hacia personas y comunidades de estos pueblos son menos habituales que tiempo atrás, éstos aún persisten.

No obstante, el racismo no sólo se expresa y ejerce mediante comportamientos "visibles", que es a los que suele limitarse la aplicación de la expresión "discriminación racial". También opera a través de desventajas acumuladas a lo largo de siglos, cuya existencia ha sido "naturalizada". Se trata de inequidades, desigualdades y exclusiones que se reproducen y multiplican mediante creencias, prejuicios, formas de "sentido común", normas, dispositivos y prácticas institucionales. A este complejo conjunto de factores se suele hacer referencia mediante la idea de "racismo estructural".

Las instituciones y políticas educativas no escapan a estos problemas, sino que son parte de ellos. A lo largo de la historia, los Estados coloniales y poscoloniales han utilizado la educación

\footnotetext{
${ }^{1}$ La expresión "América Latina" no formaba parte del léxico de los movimientos independentistas de comienzos del siglo xix. La idea de "latinidad" y su aplicación como un adjetivo fue propuesta en 1836 por el intelectual francés Michel Chevalier. "América Latina" como un nombre compuesto apareció por primera vez en un libro del intelectual colombiano José María Torres Caicedo en 1865 (Ardao, 1980).
} 
como un medio para afirmar y legitimar su dominio sobre los pueblos indígenas y afrodescendientes. Sin embargo, desde hace varias décadas, en un número creciente de universidades y otras instituciones de educación superior (IES) de América Latina se han desarrollado posiciones críticas al respecto. Algunas de ellas van más allá de lo argumentativo y orientan prácticas concretas en los ámbitos de gestión, docencia, investigación y vinculación social. ${ }^{2}$ Este texto busca aportar al análisis de los retos que enfrentan estas prácticas, con el propósito de contribuir a su fortalecimiento.

Palabras clave: Racismo; América Latina; discriminación; exclusión; racismo estructural; políticas educativas; Estado colonial; Estado poscolonial; vinculación social; responsabilidad social universitaria.

\begin{abstract}
Racism is an ideology according to which human beings would be classified in "races", where some of them would be superior to others. In Latin America, this ideology, which was originated in the colonial period, still permeates the way of looking at the world from a large part of the population, and its consequences particularly affect the individuals and communities of indigenous and afro-descendant peoples.

While in some "Latin American" countries (an expression which at least silences the existence of indigenous and afro-descendant peoples) overtly discriminatory behaviors towards individuals and communities of these peoples is less common than in the past, they persist.

However, racism is not only expressed and exercised through "visible" behavior, which is often limited to the application of the expression "racial discrimination". It also operates through disadvantages accumulated over centuries, whose existence has been "naturalized". These are inequities and forms of inequality and exclusion that are reproduced and multiplied through both beliefs, prejudices, and forms of "common sense", as well as institutional norms, arrangements, and practices. This complex set of factors is often referred to as "structural racism".

Educational institutions and policies do not escape these problems but are part of them. Throughout history, colonial and post-colonial states have used education as a means of affirming and legitimizing their dominance over indigenous and afro-descendants people. But for several decades, an increasing number of universities and other higher education institutions (HEI) in Latin America have been developing critical positions regards. Some of them go beyond argumentative and guide concrete practices in the areas of management, teaching, research, and social engagement. This text seeks to contribute to the analysis of the challenges faced by these practices, with the aim of contribute to their strengthening.

Keywords: Racism; Latin America; Discrimination; Exclusion; Structural Racism; Educational Policies; Colonial State; Post-Colonial State; Social Engagement; University Social Responsibility.
\end{abstract}

Fecha de recepción: 25/10/2019

Fecha de aceptación: 28/01/2020

\footnotetext{
${ }^{2}$ En este texto, como en otros anteriores, utilizo la expresión "vinculación social" de manera genérica para hacer referencia a lo que en diferentes ámbitos institucionales y orientaciones de trabajo, según los casos, suele nombrarse como "extensión universitaria" (expresión que en algunos casos se acompaña de adjetivaciones como "de doble vía" u otras), "investigación-acción" (en algunos casos planteada además como "participativa"), "aprendizaje servicio" (en algunos casos especificado como "solidario"), "diálogo de saberes", "voluntariado" y "responsabilidad social universitaria”, entre otras modalidades de acción universitaria (Mato, 2015).
}

70 - Los desafios del racismo a la gestión, docencia, investigación y vinculación social en la educación superior

Daniel Mato. DIDAC 76 (2020): 69-76 


\section{Racismo y educación superior}

A lo largo de la historia, las universidades y otras instituciones de educación superior (IES) "han jugado papeles importantes tanto en el proceso de construcción de representaciones homogeneizadoras de las poblaciones de los respectivos países, como en la transformación de los pueblos indígenas y afrodescendientes en objetos de estudio, incluso en contra de su voluntad" (Mato, 2018b). Lo han hecho desde enfoques etnocéntricos de investigación que produjeron representaciones descalificadoras de sus "razas", formas de vida, visiones de mundo, proyectos de futuro, conocimientos y modos de producción de los mismos.

Avanzar hacia la erradicación del racismo en la educación superior resulta clave para lograr lo mismo en todos los ámbitos de cada una de las respectivas sociedades, pues en este nivel educativo se forman técnicos y profesionales que ocupan posiciones sociales, políticas y económicas de gran incidencia.

Entre otros profesionales, en las universidades y otras IES se gradúan docentes que juegan papeles clave en todos los niveles de los sistemas educativos. A esto se agrega que ellas mismas constituyen espacios de formación ciudadana y de opinión pública.

Por fortuna, desde hace ya varias décadas, en un número creciente de universidades y otras IEs de América Latina se han desarrollado diversas corrientes críticas del cuestionable papel que juega la educación superior en la historia de las sociedades latinoamericanas. Algunas de ellas se expresan principalmente a través de formulaciones argumentativas. Otras van más allá y llevan adelante variadas modalidades de intervención social e institucional, en las que se articulan prácticas de gestión, docencia, investigación y vinculación social deliberadamente orientadas a revertir esa lamentable historia.

Se trata de un numeroso conjunto de experiencias muy diversas entre sí, algunas de las cuales se desarrollan en el marco de instituciones "convencionales"; otras, en el de instituciones interculturales, y otras más en el de instituciones "propias" de organizaciones indígenas o afrodescendientes.
Por los límites de extensión de este texto, no es posible exponer aquí las características de estas experiencias, sus avances y desafíos, y las relaciones de colaboración que crecientemente sostienen entre sí. No obstante, estos asuntos han sido analizados en algunas publicaciones colectivas (Baronnet \& Bermúdez Urbina, 2019; CGEIB, 2004; Di Caudo et al., 2016; Hernández Loeza et al., 2013; Mato, 2008, 2009, 2015, 2016, 2017, 2018a, 2019).

\section{Diversidad cultural, racismo e interculturalidad en la Declaración de la III Conferencia Regional de Educación Superior (CRES)}

Las Conferencias Regionales de Educación Superior (CRES) constituyen el mecanismo de concertación de recomendaciones de políticas para este nivel educativo más ambicioso e influyente con el que se cuenta en América Latina y el Caribe.

Hasta la fecha se han realizado tres de estas conferencias. La primera de ellas se llevó a cabo en La Habana, Cuba, en 1996; la segunda en Cartagena de Indias, Colombia, en 2008, y la tercera en Córdoba, Argentina, en 2018. Las tres han reunido a las autoridades de educación superior de todos los gobiernos de la región, así como a las autoridades de buena parte de las universidades de estos países, especialistas en el tema, dirigentes de organizaciones gremiales y estudiantiles. Cada una de estas reuniones produjo un documento final de carácter declarativo y un conjunto de recomendaciones.

En reconocimiento de los problemas que afectan a la educación superior comentados en la sección anterior de este texto, como también de los avances mencionados, la declaración final de la II CREs destacó los problemas derivados del carácter monocultural de la educación superior y la necesidad de avanzar en los tipos de transformaciones que ya se venían adelantando a través de las experiencias comentadas. Sin embargo, el documento final de la III CRes fue más lejos y además enfatizó la necesidad de erradicar el racismo y la discriminación racial (Mato, 2018a).

Por su importancia, conviene citar un extracto de las principales recomendaciones de la Declaración de la III CREs: 
Las políticas y las instituciones de educación superior deben contribuir proactivamente a desmontar todos los mecanismos generadores de racismo, sexismo, xenofobia, y todas las formas de intolerancia y discriminación. Resulta imperativo que se garanticen los derechos de todos los grupos de población discriminados por raza, etnicidad, género, situación socioeconómica, discapacidad, orientación sexual, religión, nacionalidad y desplazamiento forzado.

Es necesario promover la diversidad cultural y la interculturalidad en condiciones equitativas y mutuamente respetuosas. El reto no es sólo incluir en las instituciones de educación superior a mujeres, personas con discapacidad, miembros de pueblos indígenas y afrodescendientes e individuos de grupos sociales históricamente discriminados, sino transformarlas para que sean social y culturalmente pertinentes.

Estos cambios deben asegurar la incorporación en las instituciones de educación superior de las cosmovisiones, valores, conocimientos, saberes, sistemas lingüísticos, formas de aprendizaje y modos de producción de conocimiento de dichos pueblos y grupos sociales.

Es ineludible reconocer y valorar las epistemologías, modos de aprendizaje y diseños institucionales propios de pueblos indígenas y afrodescendientes, comunidades campesinas y otras socioculturalmente diferenciadas.

\section{$[\ldots]$}

Existe una importante deuda histórica de los Estados y sociedades latinoamericanas y caribeñas con los pueblos indígenas y afrodescendientes. Si bien sus derechos son reconocidos en numerosos instrumentos internacionales y en la mayoría de las constituciones nacionales, existe un alarmante déficit en el disfrute efectivo de los mismos, incluso en la educación superior. Las instituciones de educación superior deben garantizar el disfrute efectivo de estos derechos y educar a la población en general, y particularmente a sus comunidades, contra el racismo y todas las formas de discriminación y procedimientos conexos de intolerancia.

Es fundamental promover y facilitar el aprendizaje de las lenguas de pueblos indígenas y afrodescendientes, así como su uso efectivo en las prácticas de docencia, investigación y extensión. La formación de docentes interculturales bilingües para todos los niveles de los sistemas educativos es un deber prioritario de la educación superior (recuperado de http://espacioenlaces. org/wp-content/uploads/2018/07/declaracion_ cres2018.pdf).

El reto ahora es llevar esas recomendaciones a la práctica en todas las universidades y otras IEs de América Latina. Esto entraña importantes desafíos para todos los actores y funciones de los sistemas de educación superior, y además demanda analizar las diversas formas en que el racismo afecta a tales sistemas.

\section{Las diversas modalidades del racismo en la educación superior}

Para avanzar hacia la erradicación del racismo en la educación superior no sólo es necesario diferenciar entre racismo y discriminación racial, con el perti-

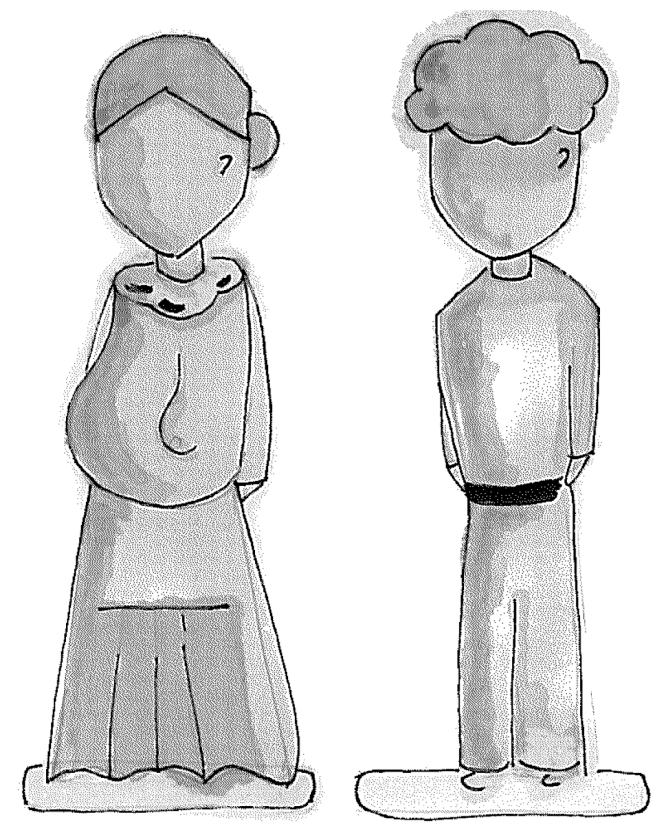

72 - Los desafios del racismo a la gestión, docencia, investigación y vinculación social en la educación superior 
nente pero limitado propósito de evitar esta última. Además, es menester afinar el análisis de lo que suele llamarse "racismo estructural", desagregar analíticamente este concepto, con el fin de diseñar acciones eficaces para superarlo.

Para esto resulta útil diferenciar entre factores que operan en diversos ámbitos. Existen algunos que, vistos desde los sistemas de educación superior, pueden considerarse socialmente estructurales, en el sentido de que afectan a la sociedad en su conjunto, es decir, que no son específicos de su ámbito sistémico. A su vez, existen otros factores de carácter sistémico, en tanto que afectan a las políticas y los diseños de cada sistema de educación superior. Por último, existen otros que son de naturaleza institucional, es decir, que caracterizan a los diseños y las prácticas institucionales, las de cada universidad o IES en particular. Desde luego, estas diferenciaciones analíticas no deben hacernos perder de vista que todos ellos se encuentran articulados, así como que se actualizan y reproducen a través de las prácticas de actores sociales concretos, dentro y fuera del campo de la educación superior.

Para los propósitos de este análisis y del diseño de estrategias de intervención, resulta útil detallar que los factores socialmente estructurales son aquellos que corresponden al contexto social en sentido amplio, más allá del ámbito del sistema de educación superior. Ejemplos de este tipo de factores son las desventajas que provienen del despojo de los territorios de los pueblos indígenas iniciado en los tiempos de la invasión y colonización europea, y continuado en las repúblicas poscoloniales, que privaron a comunidades de pueblos indígenas de sus fuentes de alimentos y cobijo. Estos problemas continúan hasta nuestros días a través de explotaciones agrícolas y mineras, desarrollos urbanísticos y turísticos, y otras actividades que además conducen a la destrucción o contaminación de ríos y humedales, además de la penetración de sus territorios. Problemas semejantes también afectan a las comunidades afrodescendientes que tras huir de la esclavitud, o culminada ésta, establecieron espacios territoriales de subsistencia (quilombos, cumbés o palenques, entre otras deno- minaciones, en diversos países), donde constantemente sufren la amenaza de ser expulsados o que son afectados por daños ambientales.

Unas y otras comunidades continúan sufriendo este tipo de problemas a lo largo y ancho de América Latina. Los escasos avances en el reconocimiento de derechos territoriales de estos pueblos generalmente resultan insuficientes para asegurar la sostenibilidad de sus modos de vida. Además, la defensa de esos derechos frente a la voracidad y la violencia de sectores económicos poderosos suelen demandar acciones permanentes por parte de las respectivas comunidades, las cuales con frecuencia son criminalizadas por los Estados (onU, 2002, 2005).

Estos factores, sumados a problemas sanitarios, dificultades de acceso a la justicia, servicios de salud y educación de calidad, suelen motivar desplazamientos forzosos de estas poblaciones o miembros de ellas hacia centros urbanos. Su inserción en estos contextos suele darse en condiciones aún más desventajosas que las que en ellos padecen otros sectores sociales afectados por condiciones de precariedad y pobreza económica. Esto por lo general se debe a su condición de migrantes relativamente carentes de conocimientos prácticos acerca de sus nuevos lugares de residencia, así como de redes sociales de apoyo, por diferencias culturales y a veces lingüísticas.

Estos procesos complejos y de larga evolución histórica tienen diversos tipos de consecuencias, pero en lo referente al campo educativo se expresan, por ejemplo, en que las tasas de analfabetismo, como también las de educación primaria o secundaria incompleta, registran valores más elevados entre la población indígena y afrodescendiente que los correspondientes a otros sectores de población de cada uno de los países de la región. En este sentido, estos tipos de problemas generan inequidad y exclusión, y constituyen causas estructurales de la escasa participación de personas indígenas y afrodescendientes entre el estudiantado, docentes, autoridades y funcionarios de las instituciones de educación superior.

Lo alarmante del caso es que la existencia de estos tipos de problemas ha sido "naturalizada" por buena parte de las respectivas sociedades. A esta in- 
visibilización contribuyen significativamente los sistemas educativos y los medios de comunicación masiva, sea ocultando estos problemas o difundiendo representaciones de los mismos que acaban justificándolos desde matrices discursivas racistas. Además, estos problemas no suelen ser objeto de políticas públicas específicas y cuando lo son, éstas suelen resultar ineficaces, por problemas de diseño, ejecución o presupuesto.

Es relativamente poco lo que se puede hacer de manera directa e inmediata desde las universidades y otras IES para revertir estos problemas. En cambio, se puede contribuir desde una perspectiva de más largo aliento, a través de la formación que estas instituciones brindan. Por ejemplo, se podrían dar a conocer tales procesos históricos y contemporáneos a los cursantes de todas las carreras. Asimismo, es posible dedicar iniciativas de investigación al estudio de estos problemas. También se pueden desarrollar experiencias de vinculación social universitaria que estén expresamente orientadas a fortalecer a las comunidades afectadas y, junto con ellas, a las instituciones educativas de nivel primario y secundario que las atienden. Estos últimos tipos de acciones, pueden ser impulsadas desde la gestión y, de este modo, establecer programas de acción afirmativa que estimulen y favorezcan el ingreso, exitosa trayectoria y graduación de estudiantes de esos pueblos, así como la incorporación de docentes y funcionarios de estos mismos orígenes.

Desde hace tiempo, algunas universidades y otras IES de varios países de la región ya desarrollan valiosas iniciativas semejantes a las mencionadas. Sin embargo, suelen hacerlo con escasos recursos, sin exigir de manera categórica a los gobiernos los presupuestos necesarios para sostenerlas. En este sentido, parece existir una omisión grave: tanto los gobiernos como las universidades y otras IES parecen olvidar que las oportunidades educativas a las personas de estos pueblos —en todos los niveles y en condiciones equitativas, y que resulten apropiadas a sus con- textos e intereses - constituyen derechos establecidos en múltiples convenios internacionales, así como en las constituciones nacionales y las leyes de la mayoría de los países de la región. ${ }^{3}$

Avanzar en esta dirección puede resultar más viable si, además de enfocarse en los problemas socialmente estructurales, se procura resolver los de carácter sistémico e institucional. Mientras se exige a los gobiernos que cumplan con sus deberes constitucionales, es posible avanzar a escalas más accesibles desde las prácticas de gestión, docencia, investigación y vinculación social.

Los factores sistémicos que aseguran la reproducción del racismo no son menores, y al menos algunos de ellos pueden resolverse con inteligencia y creatividad institucional. Por ejemplo, las normativas vigentes en los sistemas de educación superior de todos los países de la región impiden que especialistas altamente calificados en ciertos temas den clases en instituciones de este nivel si carecen de título universitario. Este tipo de normas impide que miembros de pueblos indígenas y afrodescendientes — que son grandes conocedores, por ejemplo, de usos terapéuticos de especies vegetales, mejoramiento de semillas o manejo ambiental- den clases en las universidades y otras IES. Y cuando se les permite dar clases, estas normas también causan que no sean reconocidos y remunerados bajo las mismas condiciones de quienes poseen títulos universitarios. Esta miopía sistémica contrasta con la búsqueda de tales conocimientos, y de especialistas en ellos, por parte de corporaciones farmacéuticas y agroindustriales. Algo semejante, y en realidad aún más grave, ocurre con los mejores hablantes de las lenguas de pueblos indígenas. Gracias a iniciativas de docentes e investigadores conocedores del valor de esos especialistas, del apoyo de funcionarios y autoridades, en algunas universidades y otras IES se ensayan soluciones a dichos problemas sistémicos.

Normativas y culturas sistémicas e institucionales han constreñido los espacios de aprendizaje de muchas carreras al trabajo en el aula, desperdiciando

\footnotetext{
${ }^{3}$ Véanse especialmente: Organización de las Naciones Unidas. (1967). Convención Internacional sobre la Eliminación de Todas las Formas de Discriminación Racial. Recuperado de https://www.ohchr.org/sp/ProfessionalInterest/Pages/CERD.aspx; Organización Internacional del Trabajo. (1989). Convenio sobre pueblos indígenas y tribales (núm. 169). Recuperado de https://www.ilo.org/dyn/normlex/ es/f?p=NORMLEXPUB:12100:0::NO::P12100_ILO_CODE:C169
}

74 - Los desafíos del racismo a la gestión, docencia, investigación y vinculación social en la educación superior Daniel Mato. DIDAC 76 (2020): 69-76 
así valiosas oportunidades que se presentan fuera de ellas. En algunas carreras resulta casi inconcebible graduarse sin desarrollar prácticas en espacios sociales extrauniversitarios. Las prácticas de agronomía son tal vez el mejor ejemplo de este tipo, aunque desde luego también es posible hacerlas transitando entre aulas y laboratorios, sin jamás "ensuciarse" los zapatos con la tierra del campo.

Los procesos de aprendizaje en la práctica y mediante la resolución de problemas son vitales en la formación de profesionales de la medicina y la odontología. Aunque en estos casos, las prácticas muchas veces se realizan sólo en espacios "académicamente" controlados, incluso aunque no sean aulas, sino consultorios, laboratorios o quirófanos. Sin embargo, en ciertas especialidades de estas carreras en algunas universidades se procura complementar la formación con experiencias en campo, así como en algunos países el llamado "servicio social" es requisito para graduarse de estas carreras. En un buen número de casos, estas actividades se realizan en comunidades de pueblos indígenas y afrodescendientes.

Aun así, esto no supone que se hayan resuelto los problemas de racismo. Por el contrario, dadas las pautas de trabajo, en muchos casos acaban reforzándolo, pero en otros, estas experiencias abren caminos de conocimiento, sensibilización y compromiso. Es necesario fortalecer estos tipos de experiencias positivas y capacitar tanto a los docentes como a los estudiantes de estas carreras para asegurar que todas las experiencias de este tipo, y no sólo algunas, resulten positivas para la erradicación del racismo. Los caminos que se han construido para erradicar los problemas de acoso, discriminación y violencia de género en las universidades y otras IEs pueden resultar inspiradores para hacer lo propio respecto del racismo y la discriminación racial.

Curiosamente, en las carreras de humanidades y ciencias sociales es menos frecuente que se busque implementar espacios de aprendizaje fuera de las aulas y más allá de las lecturas, en comparación con las formaciones profesionales antes referidas. No obs- tante, el desarrollo de experiencias de aprendizaje en campo y en la práctica, así como el trabajo en colaboración con diversos tipos de comunidades (incluidos los pueblos indígenas y afrodescendientes), constituyen modalidades de trabajo que están ganando terreno desde hace varias décadas en numerosas carreras humanistas y de ciencias sociales. Sin embargo, con frecuencia estas formas innovadoras de aprendizaje (innovadoras no sólo desde un punto de vista técnico, sino también ético y sensible) suelen ser desarrolladas un tanto a contramano de las normativas y planes de estudio. Estilan demandar cargas de trabajo extra para docentes y estudiantes que no son reconocidas por las instituciones; tales son típicamente los problemas que enfrentan las iniciativas de vinculación social universitaria (Mato, 2015). ${ }^{4}$

El caso es que para avanzar en la formación de profesionales y técnicos "libres de racismo" y capaces de actuar como agentes sociales que contribuyan proactivamente a erradicar el racismo, este tipo de formación, más allá de la academia, estar en contacto directo con comunidades indígenas y afrodescendientes, como también con otros sectores sociales históricamente discriminados, resulta imprescindible. Avanzar en esta dirección en las universidades y otras IEs es más accesible que acabar con los factores de racismo socialmente estructural. Pero, además, es condición para ello.

Si no logramos erradicar el racismo en la educación superior, difícilmente logremos hacerlo en cada una de nuestras sociedades en su conjunto. Por eso, la declaración final de la III CRES, entre otras recomendaciones, taxativamente encomendó:

Las instituciones de educación superior deben garantizar el disfrute efectivo de estos derechos [los de pueblos indígenas y afrodescendientes] y educar a la población en general, y particularmente a sus comunidades, contra el racismo y todas las formas de discriminación y formas conexas de intolerancia (recuperado de http://espacioenlaces.org/wp-content/uploads/2018/07/ declaracion_cres2018.pdf).I

\footnotetext{
${ }^{4}$ Considero conveniente recordar que, como fue mencionado en la nota 2, utilizo esta expresión de manera amplia y abarcadora de extensión, investigación-acción, voluntariado y otras.
} 
Ardao, A. (1980). Génesis de la idea y el nombre de América Latina. Caracas: Centro de Estudios Latinoamericanos Rómulo Gallegos.

Baronnet, B. \& Bermúdez Urbina, F. M. (Coords.). (2019). La vinculación comunitaria en la formación de profesionales indígenas en México. México: ANuIEs.

Caudo, M. V. di, Llanos Erazo, D. \& Ospina Alvarado, M. C. (Coords.). (2016). Interculturalidad y educación desde el sur. Contextos, experiencias y voces. Quito: Abya Yala y Universidad Politécnica Salesiana.

Coordinación General de Educación Intercultural Bilingüe (СGEIB). (Comp.). (2004). Educación superior para los pueblos indígenas de América Latina. Memorias del Segundo Encuentro Regional. México: CGEIB, SEP.

Hernández Loeza, S., Ramírez Duque, M. I., Manjarrez Martínez, Y. \& Flores Rosas, A. (Coords.). (2013). Educación intercultural a nivel superior. Reflexiones desde diversas realidades latinoamericanas. Huehuetla (Puebla, México): Universidad Intercultural del Estado de Puebla.

Mato, D. (2015). Vinculación social universitaria en Argentina. Diversidad de orientaciones de trabajo, logros y dificultades de las experiencias apoyadas por el Programa Nacional de Voluntariado Universitario. CPU-e, Revista de Investigación Educativa, 20, 132-149.

Mato, D. (2018). Diversidad cultural e interculturalidad en la III Conferencia Regional de Educación Superior - CRES 2018. Integración y Conocimiento, 7(2), 37-61.

Mato, D. (Coord.). (2008). Diversidad cultural e interculturalidad en Educación Superior. Experiencias en América Latina. Caracas: Unesco-IESALC.

Mato, D. (Coord.). (2009). Instituciones Interculturales de Educación Superior en América Latina. Procesos de Construcción, Logros, Innovaciones y Desafíos. Caracas: Unesco-IESALC.
Mato, D. (Coord.). (2015). Educación superior y pueblos indigenas. Contextos y Experiencias. Sáenz Peña, Buenos Aires: EDUNTREF.

Mato, D. (Coord.). (2016). Educación superior y pueblos indígenas en América Latina. Experiencias, interpelaciones y desafios. Sáenz Peña, Buenos Aires: eduntref y México: unam.

Mato, D. (Coord.). (2017). Educación superior y pueblos indigenas en América Latina. Politicas y prácticas de inclusión, democratización e interculturización. Sáenz Peńa, Buenos Aires: eduntref y José María Morelos, Q. R., México: UIMQROo.

Mato, D. (Coord.). (2018a). Educación superior y pueblos indigenas en América Latina. Políticas y experiencias de inclusión y colaboración intercultural: Sáenz Peña, Buenos Aires: EDUNTREF.

Mato, D. (2018b). Educación superior y pueblos indígenas: experiencias, estudios y debates en América Latina y otras regiones del mundo. Revista del Cisen Tramas/ Maepova, 6 (2), 41-65.

Mato, D. (Coord.). (2019). Educación superior y pueblos indigenas en América Latina. Colaboración intercultural: experiencias y aprendizajes. Sáenz Peña, Buenos Aires: eduntref.

Organización de Naciones Unidas (ONU). 2002. Declaración y Programa de Acción de la Conferencia Mundial contra el Racismo, la Discriminación Racial, la Xenofobia y las Formas Conexas de Intolerancia. Recuperado de https:// www.ohchr.org/Documents/Publications/Durban_text_ en.pdf

Organización de Naciones Unidas (ONU). 2005. Report of the Special Rapporteur on the Situation of Human Rights and Fundamental Freedoms of Indigenous People, Rodolfo Stavenhagen (E/CN.4/2005/88). Recuperado de https:// www.right-docs.org/doc/a-hrc-4-32/

\section{Semblanza}

Doctor en Ciencias Sociales por la Universidad Central de Venezuela (UCV), 1990.

Desde 2010 es Investigador Principal del Consejo Nacional de Investigaciones Científicas y Técnicas - CONICET, con sede en la Universidad Nacional de Tres de Febrero UNTREF, Argentina.

Es Director de la Cátedra UnEsco Educación Superior y Pueblos Indígenas y Afrodescendientes en América Latina y Director Adjunto del Centro Interdisciplinario de Estudios Avanzados (CIEA).

Entre 2007 y 2018 dirigió el Proyecto Diversidad Cultural e Interculturalidad del Instituto Internacional de la unESCO para la Educación Superior en América Latina y el Caribe UNESCO-IESALC.

Desde 1986 desarrolla diversas experiencias de trabajo en colaboración con intelectuales y organizaciones e indígenas y afrodescendientes de varios países latinoamericanos.

Entre 1978 y 2010 fue docente e investigador de la Universidad Central de Venezuela.

Ha sido profesor visitante en universidades de varios países latinoamericanos, España y Estados Unidos.

76 - Los desafios del racismo a la gestión, docencia, investigación y vinculación social en la educación superior Daniel Mato. DIDAC 76 (2020): 69-76 